\title{
Downregulation of claudin-7 potentiates cellular proliferation and invasion in endometrial cancer
}

\author{
XIAOCUI LI ${ }^{1 *}$, YUHONG LI $^{1 *}$, HAIFENG QIU ${ }^{2}$ and YUDONG WANG ${ }^{1}$ \\ ${ }^{1}$ Department of Gynecology, International Peace Maternity and Child Health Hospital, Shanghai Jiaotong University, \\ Shanghai 200030; ${ }^{2}$ Department of Obstetrics and Gynecology, First People's Hospital, \\ Shanghai Jiaotong University, Shanghai 200030, P.R. China
}

Received December 19, 2012; Accepted March 27, 2013

DOI: $10.3892 / 01.2013 .1330$

\begin{abstract}
Claudin-7, a tight junction protein, has been demonstrated to be abnormally regulated in several types of human cancer. The present study aimed to investigate the expression and function of claudin-7 in endometrial cancer. In total, 31 pairs of endometrial cancer samples and their adjacent normal tissues were used to detect the expression of claudin-7 by immunohistochemical staining. Compared with the corresponding normal tissues, $45.2 \%$ of the endometrial cancer tissues exhibited weak or absent claudin-7 protein expression. Low levels of claudin-7 were correlated with a late tumor stage $(\mathrm{P}=0.023)$ and low histological grade $(\mathrm{P}=0.018)$. Claudin-7 was either overexpressed in AN3CA endometrial cancer cells, via plasmid cDNA transfection, or silenced by RNA interference in Ishikawa cells. Following either type of experimental manipulation, cellular proliferation and invasion were determined by 3-(4,5-dimethylthiazol-2-yl)-2,5-diphenyltetrazolium bromide (MTT) assay, wound healing and transwell assays, respectively. The silencing of claudin-7 significantly increased cellular proliferation $(\mathrm{P}=0.032)$ and invasion $(\mathrm{P}=0.020)$ rates. Consistent with these results, the increased expression of claudin-7 decreased the proliferation $(\mathrm{P}=0.021)$ and invasiveness $(\mathrm{P}=0.012)$ of the AN3CA cells. A low expression of claudin-7 in the endometrial cancer cells was indicative of a late tumor stage and low histological grade. Additionaly, restoration of claudin-7 inhibited the proliferation and invasion of endometrial cancer cells, thus providing a potential therapeutic strategy.
\end{abstract}

Correspondence to: Professor Yudong Wang, Department of Gynecology, International Peace Maternity and Child Health Hospital, Shanghai Jiaotong University, 910 Hengshan Road, Shanghai 200030, P.R. China

E-mail: owangyudong@126.com

*Contributed equally

Key words: claudin-7, invasion, endometrial cancer

\section{Introduction}

The loss of cell-cell adhesion is a significant step in tumor cell metastasis (1). Claudins are a family of tight junction proteins that regulate cell adhesion and polarity. Thus, they appear to be significant in regulating the process of metastasis. First described in 1998, claudins contain four transmembrane domains and, to date, 24 family members have been identified. These proteins have molecular weights of 20-27 kDa and are widely expressed in the majority of epithelial cells $(2,3)$. Certain claudin family members have been identified to be abnormally regulated in several types of cancer in humans. In particular, claudin-7 was observed to be downregulated in breast cancer and head and neck squamous cell carcinoma. These findings suggest that claudin-7 may be involved in the epithelial-mesenchymal transition (EMT). Following the restoration of claudin-7 levels, cancer cells have been demonstrated to exhibit decreased motility and invasion abilities $(4,5)$.

Endometrial cancer is the primary gynecological malignancy in the majority of countries (6). In the USA, $\sim 8,010$ fatalities resulting from endometrial cancer and 47,130 new cases are predicted this year (6-8). At the time of diagnosis, $\sim 25 \%$ of patients present with regional or distant metastases (stages III or IV). Notably, the prognosis associated with this group is usually unfavorable (9). Therefore, specific strategies targeting tumor invasion should be a priority. However, detailed mechanisms governing endometrial cancer metastasis are not well defined. Previous studies have suggested that claudins may play a critical role in this process.

In endometrial cancer, several of the claudins, including claudin-1, -3 and -4 , have previously been demonstrated to be involved in maintaining tight junctions and in preventing tumor cell dispersion and invasion $(10,11)$. In the present study, the functions of claudin-7 were investigated in endometrial cancer.

\section{Materials and methods}

Immunohistochemical staining assay. Human endometrial cancer tissue microarrays, comprising 31 pairs of endometrial cancer tissues and their corresponding normal 
endometrial tissues, were purchased from Shanghai Xinchao Biotechnology (Shanghai, China). All tissues had been acquired via surgical resection. The cancer cases were classified and graded according to the criteria of the International Federation of Obstetrics and Gynecology (FIGO, 2009). This study was approved by the ethics committee of the International Peace Maternity Child Health Hospital, Shanghai Jiaotong University. In brief, the tissue sample slides were rehydrated and antigen retrieval was performed in the microwave with ethylenediaminetetraacetic acid (EDTA; pH 8.0). The slides were then incubated with primary anti-claudin-7 antibodies (1:1,000; Epitomics, Inc., Burlingame, CA, USA) overnight at $4{ }^{\circ} \mathrm{C}$. Antibody staining was visualized with 3,3'-diaminobenzidine (DAB; Invitrogen Life Technologies, Carlsbad, CA, USA). For evaluation, the following criteria were used: 0 , no expression (complete negative staining); 1 , weak expression (1-15\% positive staining); 2, moderate expression (16-49\% positive staining); and 3 , strong expression (50-100\% positive staining). Scores of 0 and 1 were defined as negative expression. All slides were independently scored by two pathologists.

Cancer cell lines and cultures. The RL95-2, Ishikawa, AN3CA and KLE endometrial cancer cell lines, were routinely cultured in Dulbecco's modified Eagle's medium (DMEM)/F12 (Gibco, Auckland, New Zealand) supplemented with $10 \%$ fetal bovine serum (Biowest, Nuaillé, France) at $37^{\circ} \mathrm{C}$ with $5 \% \mathrm{CO}_{2}$.

RNA extraction and quantitative real-time reverse transcription polymerase chain reaction $(R T-P C R)$. Total RNA was extracted using TRIzol reagent (Invitrogen Life Technologies) and reverse transcribed with the RT kit from Takara Biotechnology Co., Ltd. (Dalian, China). The primers used for claudin-7 were as follows: Forward, 5'-AGAGCACGGGGATGATGAG-3' and reverse, 5'-CACCCATGGCTATACGGGC-3'. The PCR conditions were as follows: $95^{\circ} \mathrm{C}$ for $30 \mathrm{sec}, 35$ cycles at $95^{\circ} \mathrm{C}$ for $5 \mathrm{sec}$, then $60^{\circ} \mathrm{C}$ for $30 \mathrm{sec}$. $\beta$-actin was used as an endogenous control. The relative mRNA expression was calculated using the $2^{-\Delta \Delta \mathrm{Ct}}$ comparative CT method.

Western blot analysis. The primary antibodies for claudin-7 were obtained from Epitomics, Inc. The mouse monoclonal anti-glyceraldehyde-3-phosphate dehydrogenase (GAPDH), horseradish peroxidase (HRP)-conjugated anti-rabbit and anti-mouse secondary antibodies were purchased from Boshide Biotechnology Company (Wuhan, China). The specific bands were developed with enhanced chemiluminescence (Beyotime, Shanghai, China).

Silencing claudin-7 in Ishikawa cells. Claudin-7-specific siRNA was purchased from Shanghai Genepharma Co., Ltd. (China). The cells were cultured in 6-well plates for $24 \mathrm{~h}$ prior to being transfected with Lipofectamine 2000 (Invitrogen Life Technologies), according to the manufacturer's instructions.

Overexpressing claudin-7 in AN3CA cells. The plasmid pcDNA3.1-claudin-7 was purchased from Genearray Biotech
Company (Shanghai, China) and verified by sequence analysis. The AN3CA cells were transfected with pcDNA3.1-claudin-7 or an empty vector using Lipofectamine 2000. Subsequently, real-time PCR and western blot analysis were performed to verify the changes in claudin-7 expression.

Proliferation assay. The proliferation of the Ishikawa and AN3CA cells was determined by 3-(4,5-dimethylthiazol-2-yl)-2,5-diphenyltetrazolium bromide (MTT) assay. Briefly, $4 \times 10^{3}$ cells were seeded in each well of a 96-well plate and incubated overnight. At the appropriate time (48 and $72 \mathrm{~h}$ ), the cells were incubated with $10 \mu \mathrm{l}$ MTT ( $5 \mathrm{mg} / \mathrm{ml}$; Sigma, St. Louis, MO, USA) for $4 \mathrm{~h}$. Formazan crystals were subsequently dissolved in $100 \mu \mathrm{l}$ dimethylsulfoxide (DMSO; Sigma). The absorbance of the solution was measured at $570 \mathrm{~nm}$. All measurements were repeated in triplicate.

Transwell assay. The transwell chamber system (Applied Biosystems, Foster City, CA, USA) was used to investigate cellular invasive abilities. Briefly, $1 \times 10^{4}$ Ishikawa or AN3CA cells were seeded into the upper chamber. Subsequently, $500 \mu 1$ conditioned medium was added to the bottom chamber. Following $24 \mathrm{~h}$ of incubation, the cells that had migrated to the bottom membrane were stained and counted under a microscope. This experiment was performed in triplicate.

Statistical analysis. $\mathrm{P}<0.05$ was considered to indicate a statistically significant difference. SPSS software, version 16.0, (SPSS Inc., Chicago, IL, USA) was used for the statistical analysis. The analysis was performed using either a $\chi^{2}$ test or a t-test.

\section{Results}

Claudin-7 expression in human endometrial cancer. Compared with the corresponding normal endometrial tissues, the endometrial cancer tissues exhibited significantly downregulated claudin-7 expression (Fig. 1, P<0.01). In the cancer tissues, approximately half of the patients $(14 / 31,45.2 \%)$ demonstrated a loss or the total negative expression of claudin-7. A real-time RT-PCR analysis of the KLE, RL 95-2 and Ishikawa cells revealed that these cell lines positively expressed claudin-7, and that by contrast, the AN3CA cells were claudin-7-negative (Fig. 1).

Correlations between claudin-7 expression and clinicopathological characteristics. According to the statistical analysis, the reduced expression of claudin-7 was significantly correlated with the tumor stage $(\mathrm{P}=0.023)$ and histological grade $(\mathrm{P}=0.018)$, but not with the remaining characteristics that were examined (Table I).

Correlations between claudin-7 expression and other immunohistochemical parameters. No correlation was observed between claudin-7 expression and ER, PR or p53 expression $(\mathrm{P}=0.125, \mathrm{P}=0.318$ and $\mathrm{P}=0.266$, respectively; Table $\mathrm{I})$.

Claudin-7 silencing increases the proliferation and invasion of Ishikawa cells. RNA interference resulted in an $290 \%$ knockdown of claudin-7 mRNA levels in the Ishikawa cells 
A

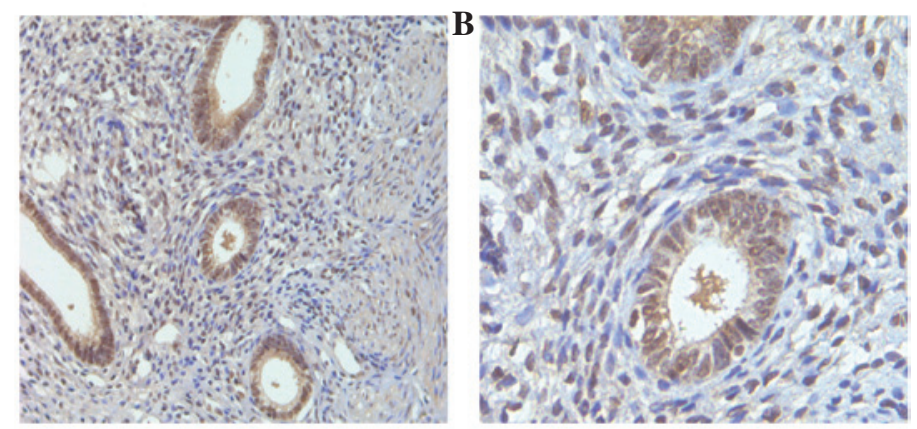

C

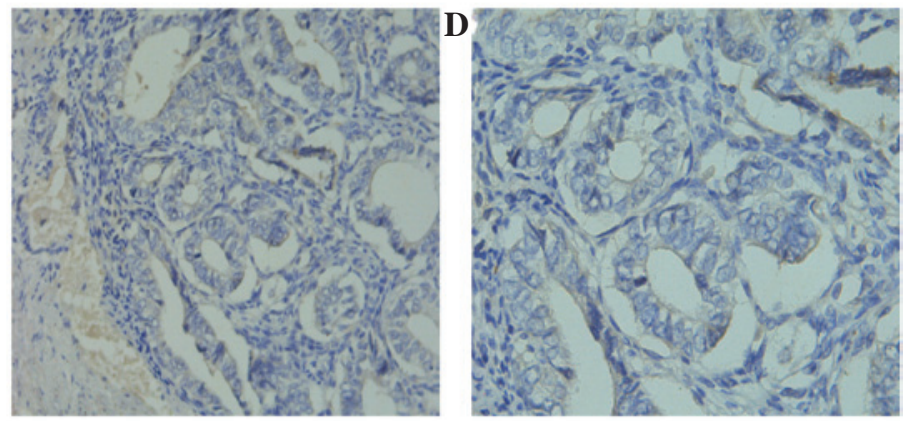

$\mathbf{E}$

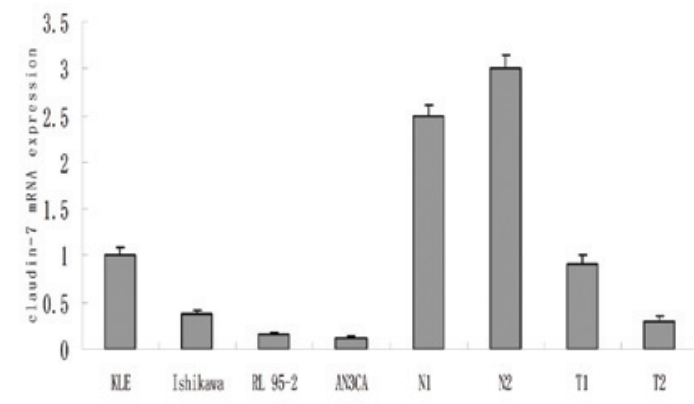

Figure 1. Immunohistochemical staining of claudin-7 expression in (A and B) normal endometrium and $(\mathrm{C}$ and $\mathrm{D})$ cancer tissues. Original magnification, (A and C) x200 and (B and D) x400; staining, DAB. (E) Claudin-7 mRNA expression in endometrial cancer cell lines, as detected by real-time reverse trancription-polymerase chain reaction analysis. N, normal tissues; T, tumor tissues; DAB, 3,3'-diaminobenzidine.

A

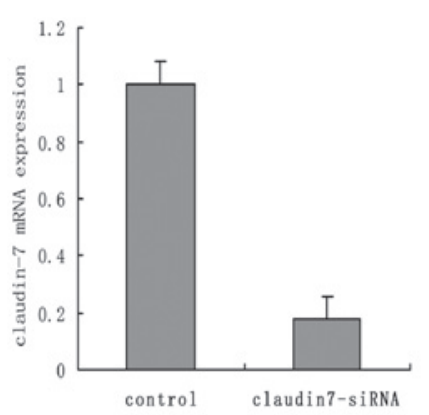

C

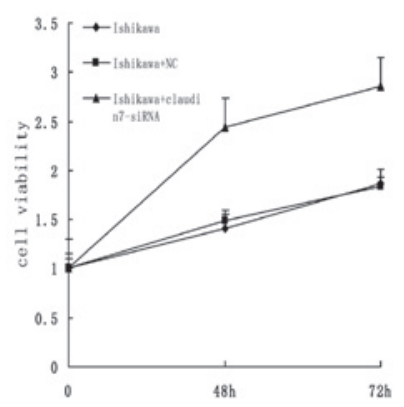

B

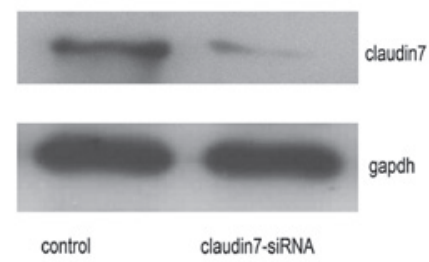

D 160

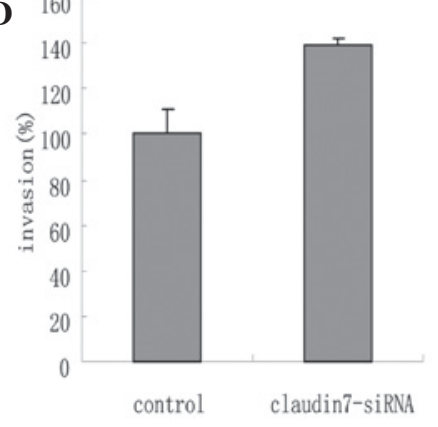

Figure 2. Downregulation of claudin-7 in Ishikawa cells and its effect on cellular growth and invasion. (A) mRNA and (B) protein expression of claudin-7 were reduced following RNA-interference. In Ishikawa cells with decreased claudin-7, cellular (C) growth and (D) invasion were all increased. NC, negative control. 
Table I. Correlations between claudin-7 expression and clinicopathological characteristics in 31 endometrial carcinoma samples.

\begin{tabular}{|c|c|c|c|c|}
\hline \multirow[b]{2}{*}{ Variables } & \multirow[b]{2}{*}{ No. of patients } & \multicolumn{2}{|c|}{ Claudin-7 expression } & \multirow[b]{2}{*}{ P-value ${ }^{a}$} \\
\hline & & Negative & Positive & \\
\hline \multicolumn{5}{|c|}{ Age (years) } \\
\hline$<60$ & 17 & 7 & 10 & \multirow[t]{2}{*}{0.715} \\
\hline$\geq 60$ & 14 & 7 & 7 & \\
\hline \multicolumn{5}{|l|}{ Stage } \\
\hline I & 21 & 9 & 12 & \multirow[t]{4}{*}{0.023} \\
\hline II & 5 & 2 & 3 & \\
\hline III & 4 & 2 & 2 & \\
\hline IV & 1 & 1 & 0 & \\
\hline \multicolumn{5}{|l|}{ Grade } \\
\hline 1 & 23 & 8 & 15 & \multirow[t]{3}{*}{0.018} \\
\hline 2 & 5 & 3 & 2 & \\
\hline 3 & 3 & 3 & 0 & \\
\hline \multicolumn{5}{|c|}{ Myometrial invasion } \\
\hline$<1 / 2$ & 19 & 9 & 10 & \multirow[t]{2}{*}{0.560} \\
\hline$\geq 1 / 2$ & 12 & 5 & 7 & \\
\hline \multicolumn{5}{|c|}{ ER expression } \\
\hline Negative & 9 & 5 & 4 & \multirow[t]{2}{*}{0.125} \\
\hline Positive & 22 & 10 & 12 & \\
\hline \multicolumn{5}{|c|}{ PR expression } \\
\hline Negative & 5 & 2 & 3 & \multirow[t]{2}{*}{0.318} \\
\hline Positive & 26 & 12 & 14 & \\
\hline \multicolumn{5}{|c|}{ p53 expression } \\
\hline Negative & 23 & 10 & 13 & \multirow[t]{2}{*}{0.266} \\
\hline Positive & 8 & 3 & 5 & \\
\hline
\end{tabular}

${ }^{\mathrm{a}}$ From the $\chi^{2}$ test.
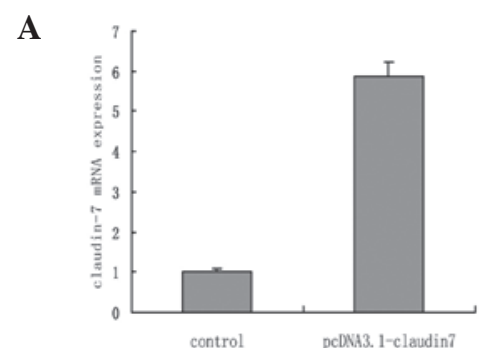

B

C

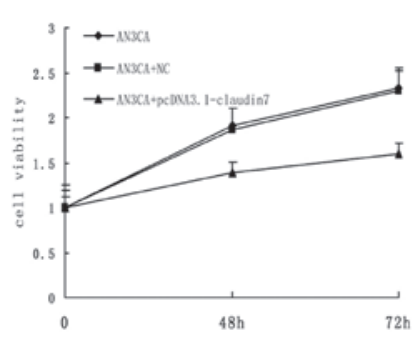

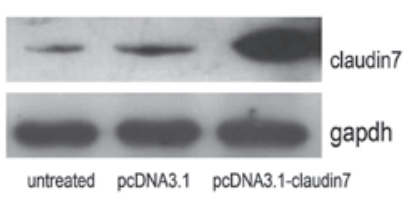

D

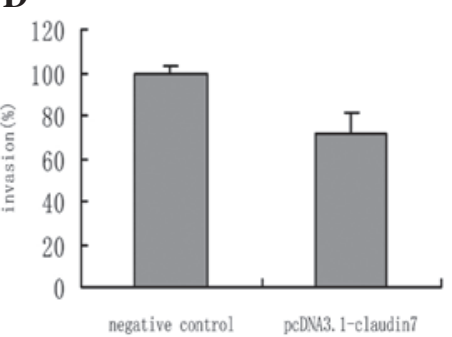

Figure 3. Upregulation of claudin-7 in AN3CA cells and its effect on cellular growth and invasion. (A) mRNA and (B) protein expression of claudin-7 was upregulated following transfection with pcDNA3.1-claudin-7. Cellular (C) growth and (D) invasion were impaired by claudin-7 overexpression. NC, negative control.

(Fig. 2A and B). The MTT assay revealed that the Ishikawa cells treated with claudin-7-siRNA possessed a higher growth rate compared with the untreated and empty vector (negative control) Ishikawa cells ( $\mathrm{P}=0.032$; Fig. $2 \mathrm{C})$. In the transwell assay, the claudin-7-silenced Ishikawa cells were more invasive than the control group ( $\mathrm{P}=0.02$; Fig. 2D). 
Claudin-7 overexpression decreases the tumorigenic properties of AN3CA cells. The transfection of the AN3CA cells with pcDNA3.1-claudin-7 resulted in the upregulation of claudin-7 at the mRNA and protein levels (Fig. 3A and B). Cellular proliferation and invasion were significantly suppressed following transfection $(\mathrm{P}=0.021$ and $\mathrm{P}=0.012$, Fig. $3 \mathrm{C}$ and $\mathrm{D}$, respectively), compared with the untreated and empty vector (negative control) AN3CA cells.

\section{Discussion}

In numerous types of human cancer, the number of cell-cell junctions decreases, permitting the escape of cancer cells from their primary sites, along with the acquisition of invasive and metastatic properties $(12,13)$. Therefore, targeting cell-cell junctions may be a valid strategy for the treatment of cancer. A number of claudins, including claudin-7, have been identified to be downregulated in various types of human cancer. Claudin-7 has been shown to be downregulated in head and neck squamous cell carcinoma, invasive ductal breast cancer and invasive esophageal cancer $(4,5,14)$. However, these studies did not analyze the manner in which claudin-7 functions to inhibit cancer cells from undergoing invasion and metastasis. Thus, the present study investigated the expression and potential mechanisms governing claudin-7 in endometrial cancer.

The study observed that claudin-7 was frequently downregulated in human endometrial cancer tissues. To investigate the mechanism by which claudin-7 affects endometrial cancer cells, claudin-7-specific siRNA was first transfected into Ishikawa cells (those demonstrating normal claudin-7 expression). The results demonstrated that invasion was significantly upregulated in the Ishikawa cells. Cellular proliferation was also significantly upregulated. Full-length claudin-7 cDNA was then cloned and overexpressed in the AN3CA cells (which were claudin-7-negative), and MTT and invasion assays confirmed similar effects.

It has been suggested that the effects of claudin-7 were generated through the attenuated activation of MAPK/ERK signaling (14). The interactions between the MAPK pathway and tight junction proteins have previously been identified. For example, the tight junction membrane protein, occludin, participates in the activation of the MAPK signaling pathway (15). In the hepatic cell lines derived from occludin-deficient mice, MAPK activation was revealed to be downregulated, triggering apoptosis and increasing claudin-2 expression. The PI3K/Akt pathway may also interact with tight junction proteins. It has been also demonstrated, in these hepatic cell lines, that the activation of Akt is decreased, while cell apoptosis is increased (16).

Overall, the present study demonstrated that claudin-7 is frequently downregulated in endometrial cancer, and that this is correlated with the tumor stage and histological grade. The ectopic expression of claudin-7 significantly regulates the proliferation and invasion of endometrial cancer cells.
Therefore, these findings may provide a potential therapeutic target for the treatment of endometrial cancer.

\section{Acknowledgements}

This study was supported by the National Natural Science Foundation of China (grant no. 81172477), the Youth Foundation of Shanghai Hygiene Bureau (grant no. 044Y06) and the Natural Science Foundation of Shanghai Science and Technology (grant no. 11ZR1440800).

\section{References}

1. Hirohashi and Kanai Y: Cell adhesion system and human cancer morphogenesis. Cancer Sci 94: 575-581, 2003.

2. González-Mariscal L, Betanzos A, Nava P and Jaramillo BE: Tight junction proteins. Prog Biophys Mol Biol 81: 1-44, 2003.

3. Morita K, Furuse M, Fujimoto K and Tsukita S: Claudin multigene family encoding four-transmembrane domain protein components of tight junction strands. Proc Natl Acad Sci USA 96: 511-516, 1999.

4. Kominsky SL, Argani P, Korz D, Evron E, Raman V, Garrett E, Rein A, Sauter G, et al: Loss of the tight junction protein claudin-7 correlates with histological grade in both ductal carcinoma in situ and invasive ductal carcinoma of the breast. Oncogene 22: 2021-2033, 2003

5. Al Moustafa AE, Alaoui-Jamali MA, Batist G, Hernandez-Perez M, Serruya C, Alpert L, Black MJ, Sladek R and Foulkes WD: Identification of genes associated with head and neck carcinogenesis by cDNA microarray comparison between matched primary normal epithelial and squamous carcinoma cells. Oncogene 21: 2634-2640, 2002.

6. Amant F, Moerman P, Neven P, Timmerman D, Van Limbergen E and Vergote I: Endometrial cancer. Lancet 366: 491-505, 2005.

7. American Cancer Society: Cancer Facts \& Figures 2011. American Cancer Society, Atlanta, 2011.

8. Siegel R, Naishadham D and Jemal A: Cancer statistics, 2012. CA Cancer J Clin 62: 10-29, 2012.

9. Dedes KJ, Wetterskog D, Ashworth A, Kaye SB and Reis-Filho JS: Emerging therapeutic targets in endometrial cancer. Nat Rev Clin Oncol 8: 261-271, 2011.

10. Pan XY, Wang B, Che YC, Weng ZP, Dai HY and Peng W: Expression of claudin-3 and claudin-4 in normal, hyperplastic, and malignant endometrial tissue. Int J Gynecol Cancer 17: 233-241, 2007.

11. Santin AD, Zhan F, Cane' S, Bellone S, Palmieri M, Thomas M, et al: Gene expression fingerprint of uterine serous papillary carcinoma: identification of novel molecular markers for uterine serous cancer diagnosis and therapy. Br J Cancer 92: 1561-1573, 2005.

12. Moldvay J, Jäckel M, Páska C, Soltész I, Schaff Z and Kiss A: Distinct claudin expression profile in histologic subtypes of lung cancer. Lung Cancer 57: 159-167, 2007.

13. Swisshelm K, Macek R and Kubbies M: Role of claudins in tumorigenesis. Adv Drug Deliv Rev 57: 919-928, 2005.

14. Lioni M, Brafford P, Andl C, Rustgi A, El-Deiry W, Herlyn M and Smalley KS: Dysregulation of claudin-7 leads to loss of E-cadherin expression and the increased invasion of esophageal squamous cell carcinoma cells. Am J Pathol 170: 709-721, 2007.

15. Lu Z, Ding L, Hong H, Hoggard J, Lu Q and Chen YH: Claudin-7 inhibits human lung cancer cell migration and invasion through ERK/MAPK signaling pathway. Exp Cell Res 317: 1935-1946, 2011.

16. Murata M, Kojima T, Yamamoto T, Go M, Takano K, Osanai M, Chiba $\mathrm{H}$ and Sawada N: Down-regulation of survival signaling through MAPK and Akt in occludin-deficient mouse hepatocytes in vitro. Exp Cell Res 310: 140-151, 2005. 